\title{
Comparison of two pivotal strategies in sparse plane rotations
}

by

Zahari Zlatev

DAIMI PB-122

July 1980

Computer Science Department AARHUS UNIVERSITY

Ny Munkegade - DK 8000 Aarhus C - DENMARK Telephone: $06-128355$

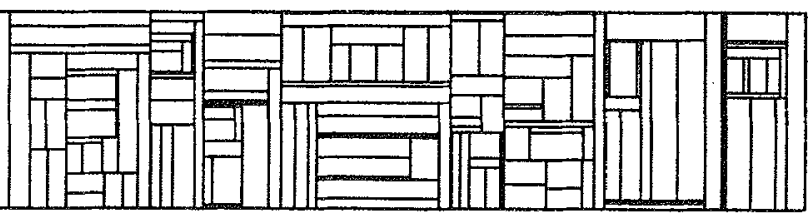




\author{
by \\ Zahari Zlatev \\ Computer Science Department \\ Aarhus University \\ Aarhus, Denmark
}

ABSTRACT Let the rectangular matrix A be large and sparse. Assume that plane rotations are used to decompose A into $R D S$ where $R^{T} R=I, \quad D$ is diagonal and $S$ is upper triangular. Both column and row interchanges have to be used in order to preserve the sparsity of matrix A during the decomposition. It is proved that if the column interchanges are fixed, then the number of non-zero elements in $S$ does not depend on the row interchanges used. However, this does not mean that the computational work is also independent of the row interchanges. Two pivotal strategies, where the same rule is used in the choice of pivotal columns, are described and compared. It is verified (by many numerical examples) that if matrix $A$ is not very sparse, then one of these strategies will often perform better than the other both with regard to the storage and the computing time. The accuracy and the robustness of the computations are also discussed. 
Let $m \in \mathbb{N}, \quad n \in \mathbb{N}, \quad b \in \mathbb{R}^{m \times 1}$ and $A \in \mathbb{R}^{m \times n}$ be given. Asume that: (i) $m \geq n$, (ii) $\operatorname{rank}(A)=n$, (iii) $n$ is large (say, $n \geq 100$ ) and (iv) A is sparse (i.e. many elements of $A$ are equal to zero). Consider the decomposition

(1.1) $\quad \mathrm{RDS}=\mathrm{PAQ}+\mathrm{E}$

where $\quad P \in \mathbb{R}^{m \times m}$ and $Q \in \mathbb{R}^{n \times n}$ are permutation matrices, $\quad E \in \mathbb{R}^{m \times n}$ is a perturbation matrix, $\mathbb{R} \in R^{m \times n}$ is such that $R^{\mathbb{T}} R=I \in \mathbb{R}^{n \times n}$, $D \in \mathbb{R}^{n \times n} \quad$ is a diagonal matrix and $S \in \mathbb{R}^{n \times n}$ is an upper triangular matrix.

Denote $\mathrm{A}_{1}=\mathrm{A}$ and assume for the moment that the diagonal matrix $D_{1} \in \mathbb{R}^{m x m}$ is given. The decomposition (1.1) is often carried out by the method of plane rotations in $n$ major steps. During the k'th major step $(k=1(1) n)$ the product $D_{k} A_{k}$ (where $D_{k} \in \mathbb{R}^{m \times m}$ is diagonal and $A_{k} \in \mathbb{R}^{m \times n}$ contains only zero elements under the diagonal in its first $k-1$ columns for $k>1$ ) is transformed into $D_{k+1} A_{k+1}$ (where $D_{k+1} \in \mathbb{R}^{m \times m}$ is diagonal and $A_{k+1} \in \mathbb{R}^{m x n}$ contains only zero elements under the diagonal in its first $k$ columns) by the following algorithm:

(i) Choose the pivotal column $j \quad(k \leq j \leq n)$ and interchange column $j$ and column $k$ if $j \neq k$. Denote the matrix so found by $\bar{A}_{k}$.

(ii) Assume that the $k^{\prime}$ th column in $\bar{A}_{k}$ has $s_{k}+1$ nonzero elements on and/or under the diagonal. Then $s_{k}$ multiplications with elementary orthogonal matrices are performed when $s_{k}>0$ so that after each multiplication one zero element in the pivotal column is produced (on or under the diagonal). 
(iii) Let the i'th row $(\mathrm{k} \leq i \leq \mathrm{m})$ be the only row which contains a non-zero element in the pivotal column after the last multiplication with an elementary orthogonal matrix auring the major step $k$. Interchange row $i$ and row $k$ if $i \neq k$.

When the computations in the n'th major step are completed, the product $D_{n+1} A_{n+1}$ will be available. It is clear that the first $n$ rows of $A_{n+1}$ form matrix $S$ and matrix $D$ from (1.1) is the upper left (nxn) - submatrix of $D_{n+1}$.

Multiplication by an elementary orthogonal matrix is often called a minor step and is carried out as follows. Consider the k'th major step again. Assume that (i) $\mu \geq k, v \geq k, \mu \neq v$ and (ii) a $\neq 0$, $a_{\nu k} \neq 0$. Then the elementary orthogonal matrix $o_{\mu \nu}$ (which differs from the identity matrix $\quad I \in \mathbb{R}^{\operatorname{mxm}}$ only by $o_{\mu \mu}=o_{\nu \nu}=\gamma \quad$ and $o_{\mu \nu}=-o_{\nu \mu}=\sigma \quad$ with $\left.\quad \gamma^{2}+\sigma^{2}=1\right)$ is used to transform into zero one of $a_{\mu k}$ and $a_{\nu k}$ It is readily seen that in fact the multiplication

(1.2) $\quad\left[\begin{array}{cc}\gamma & \sigma \\ -\sigma & \gamma\end{array}\right]\left[\begin{array}{ll}a_{\mu} & 0 \\ 0 & a_{\nu}\end{array}\right]\left[\begin{array}{llll}a_{\mu k} & \cdot & a_{\mu n} \\ a_{\nu k} & \cdot & \cdot & a_{\nu n}\end{array}\right]$

has to be performed (when $n$ is large and $A$ is sparse many of the elements of the last matrix in (1.2) are normally zero elements; therefore some sparse matrix technique, where only the non-zero elements are stored and used in the arithmetic operations, has to be implemented). In the classical Givens method $[12,13] \quad D_{k}=I \in \mathbb{R}^{m \times m}$ $(\mathrm{k}=1(1) \mathrm{n})$ and the multiplication $(1.2)$ is carried out directly, i.e. the new elements are computed by

$$
\bar{a}_{\mu j}=\gamma a_{\mu j}+\sigma a_{\nu j}, \quad \bar{a}_{\nu j}=-\sigma a_{\mu j}+\gamma a_{\nu j}, \quad j=k(1) n .
$$

This means that 2 multiplications are needed in the com- 
putation of each new element. If $D_{k}=I \quad(k=1(1) n)$ is not required, then the first two matrices in (1.2) can be refactorized, e.g. in the following way:

$(1.4) \quad\left[\begin{array}{ll}\gamma & \sigma \\ \sigma & \gamma\end{array}\right]\left[\begin{array}{ll}a_{\mu} & 0 \\ 0 & a_{\nu}\end{array}\right]=\left[\begin{array}{cc}a_{\mu}^{\gamma} & 0 \\ 0 & a_{\nu}^{\gamma}\end{array}\right]\left[\begin{array}{ll}1 & \alpha \\ \beta & 1\end{array}\right]$.

Then the multiplication

(1.5) $\quad\left[\begin{array}{ll}1 & \alpha \\ \beta & 1\end{array}\right]\left[\begin{array}{llll}a_{\mu k} & \cdot & a_{\mu n} \\ a_{\nu k} & \cdot & \cdot & a_{\nu n}\end{array}\right]$

is carried out and the new elements are computed by the formulae:

$$
\bar{a}_{\mu j}=a_{\mu j}+\alpha a_{\nu j}, \quad \bar{a}_{\nu j}=\beta a_{\mu j}+a_{\nu j}, \quad j=k(1) n .
$$

It is clear that only one multiplication per new element is needed when (1.6) are used instead of (1.3). The refactorization (1.4) is proposed by Gentieman [9] (see also [16,11]). This is not the only possible refactorization of the left-hand side of (1.4). Some other refactorizations are given by wilkinson [25], pp.12-13. In this way Wilkinson has shown that both the classical Givens method and the Gentleman version can be found as special cases in the generalized plane rotations with a suitable choice of the matrices $D_{k}$ $(k=1(1) n)$. The refactorization $(1.4)$ will be used in this paper. The following algorithm can be used in the computation of $\alpha$, $\beta$ and $\gamma$ (see also Lawson et al. [17], Björck [5] and Zlatev and Nielsen [30]). 
4.

$(1.7) \quad a_{\mu}^{2} a_{\mu k}^{2} \geq a_{\nu}^{2} a_{\nu k}^{2}$

then produce a zero element in row $v$ by

$(1.8) \quad \beta=-a_{\nu k} / a_{\mu k}, \quad \alpha=-\beta d_{\nu}^{2} / a_{\mu}^{2}$.

If

$(1.9) \quad a_{\mu}^{2} a_{\mu k}^{2}<d_{v}^{2} a_{v k}^{2}$

then produce a zero element in row $\mu$ by

$(1.10) \quad \alpha=-a_{\mu k} / a_{\nu k}, \quad \beta=-\alpha d_{\mu}^{2} / d_{\nu}^{2}$.

In both cases

$(1.11) \quad \gamma^{2}=1 /(1-\alpha \beta)$

and the new elements of the diagonal matrix $D_{k}$ involved in this minor step are computed by

(1.12) $\quad \bar{d}_{\mu}^{2}=\gamma^{2} d_{\mu}^{2}, \quad \bar{d}_{\nu}^{2}=\gamma^{2} a_{\nu}^{2}$.

Remark 1.1 It is easily seen that the above algorithm ensures that the squares of the diagonal elements of $D_{k} \quad(k=1(1) n)$ are decreased by a factor $\delta_{k i} \in\left[\frac{1}{2}, 1\right)$ after the $i$ 'th minor step $\left(i=1(1) s_{k}\right)$ of major step $k$.

$\underline{\text { Remark } 1.2}$ If $D_{k}^{2} \quad(k=1(1) n+1)$ are stored instead of $D_{k}$, 
then no square root is needed.

Remark 1.3 If the problem under consideration is not weighted, then $D_{1}=I$ can be chosen. If the problem is weighted, then $D_{1}^{2}$ must have diagonal elements equal to the squares of the weights.

If matrix $A$ is sparse, then a good choice of $P$ and $Q$. (in other words, a good pivotal strategy) will normally lead to a considerable reduction in the computing time and the storage needed. Two pivotal strategies will be considered in this paper. These pivotal strategies can be defined as follows.

Definition 1.1 The set

(1.13) $\quad \bar{C}_{k j}=\left\{a_{i j} / i=k(1) m\right\}$

is called the active part of column $j(j=k(1) n)$ at major step $k \quad(k(1) n)$.

Rule 1 At any major step $k \quad(k=1(1) n)$ the column which contains a minimal number of non-zero elements in its active part (or one of these columns if there are several) is chosen as pivotal.

\section{Definition 1.2 The set}

$(1.14) \quad \bar{R}_{k i}=\left\{a_{i j} / j=k(1) n\right\}$

is called the active part of row $i \quad(i=k(1) \mathrm{m})$ at major step k $\quad(k=1(1) n)$. 
Definition 1.3 Consider major step $k \quad(k=1(1) n)$. The number of non-zero elements in row i $(i=k(1) \mathrm{m})$ before minor step $s \quad\left(s=1(1) s_{k}\right)$ will be denoted by $r(k, s, i)$.

Rule 2 Consider major step $k \quad(k=1(1) n)$. Choose a row $i$ for which (1) $k \leq i \leq m, \quad(2) \quad a_{i k} \neq 0$ and (3) if $\mu$ is any row for which $k \leq \mu \leq \mathrm{m}$ and $a_{\mu k} \neq 0$, then $r(k, 1, i) \leq r(k, 1, \mu)$. Consider any two rows $\nu$ and $\tau \quad(k \leq \nu, \tau \leq m, \nu \neq i, \tau \neq i)$ with $a_{\nu k} \neq 0$ and $a_{\tau k} \neq 0$. The elements $a_{\nu k}$ and $a_{\tau k}$ are transformed into zero by multiplication with the elementary orthogonal matrices $o_{i \nu}$ and $o_{i \tau}$ and, moreover, if $r(k, 1, \nu)<r(k, 1, \tau)$ then avk will be transformed into zerobefore $a_{\tau k}$ (the multiplication with oiv will be performed before the multiplication with $\left.o_{i \tau}\right)$.

Rule 3 Consiaer major step k $(k=1(1) n)$. Before any minor step $s \quad\left(s=1(1) s_{k}\right)$ choose two rows $\mu$ and $v$ for which (1) $k \leq \mu, \nu \leq m, \quad$ (2) $\quad a_{\mu k} \neq 0, \quad a_{\nu k} \neq 0, \quad(3)$ if $\tau$ is any row with $k \leq \tau \leq m$ and $a_{\tau k} \neq 0$, then $\max (r(k, s, \mu), r(k, s, \nu)) \leq r(k, s, \tau)$. Produce a zero element in one of these two rows (multiplying by $o_{\mu \nu}$ ) according to the algorithm described by $(1.7)-(1.11)$.

Definition 1.4 The pivotal strategy based on Rule 1 and Rule 3 will be called Strategy 1 or a variable pivotal row strategy.

Definition 1.5 The pivotal strategy based on Rule 1 and Rule 2 will be called Strategy 2 or a fixed pivotal row strategy. 
Strategy 2 has been used by Duff [7], see also [8,21]. Strategy 1 is based on ideas suggested by Gentleman [10] and has been implemented in the sparse code described in [29,30]. A comparison of these two strategies will be carried out in this paper. The main results obtained in this comparison are:

A. If Strategy 1 and Strategy 2 are used with the same matrix Q (i.e. the column interchanges are the same), then the two strategies produce the same number of non-zero elements in matrix $S$. However, this does not mean that the computational work for obtaining the decomposition (1.1) will be the same; see Corollary 2.1 and Example 2.1 in Section 2 .

B. If matrix $A$ is very sparse and the sparsity is well preserved during the computations, then the two strategies will give similar results (especially when the number of non-zero elements in the active part of the pivotal column is less than 4 for each $k$ $(k=1(1) n) ;$ see Theorem 2.2$)$.

C. If $n \geq 100$ and if the matrix is not very sparse, then Strategy 1 will often give better results than Strategy 2 with regard both to the storage used and the computing time needed; moreover, Strategy 1 ensures more robust computations; see the numerical results in section 3 . 
8.

2. Computations with a fixed matrix Q

The following definitions and theorems are needed before the discussion of the question: what should be expected when the strategies defined in Section 1 are used with the same column interchanges (the same matrix Q )?

Definition 2.1 The set

$(2.1)$

$$
B_{k}=\left\{a_{i j} \in \bar{A}_{k} / k \leq i \leq m \quad \wedge \quad k \leq j \leq n\right\}
$$

will be called the active part of $\overline{\mathrm{A}}_{\mathrm{k}} \quad(\mathrm{k}=1(1) \mathrm{n})$.

Definition 2.2 The set

$(2.2)$

$$
L_{k i}=\left\{j / a_{i j} \neq 0 \wedge a_{i j} \in B_{k}\right\}
$$

will be called the sparsity pattern of the active part of row i $(\mathrm{i}=\mathrm{k}(1) \mathrm{m})$ at major step k $(\mathrm{k}=1(1) \mathrm{n})$.

Definition 2.3 The set

$(2.3)$

$$
M_{k j}=\left\{i / a_{i j} \neq 0 \wedge a_{i j} \in B_{k}\right\}
$$

will be called the sparsity pattern of the active part of column $j \quad(j=k(1) n)$ at major step $k \quad(k=1(1) n)$.

Definition 2.4 The set

(2.4) $\quad \mathbb{N}_{k}=\left\{j / a_{i j} \neq 0 \wedge a_{i j} \in B_{k} \wedge i \in M_{k k}\right\}$ 
will be called the union sparsity pattern of the active parts of the rows involved in the computations at major step $k \quad(k=1(1) n)$.

Remark 2.1 It is clear that

(2.5) $\quad \mathbb{N}_{k}=\bigcup_{i \in M_{k k}} L_{k i}$.

\section{Definition 2.5 The set}

(2.6) $\quad \mathbb{N}_{k j}^{*}=\left\{i / a_{i \mu} \neq 0 \wedge a_{i \mu} \in C_{k j} \wedge i \in M_{k j} \wedge k<n\right\}$

where the submatrix $\quad C_{k j} \quad$ is defined by

(2.7) $\quad C_{k j}=\left\{a_{i \mu} \in B_{k} / j \leq \mu \leq n\right\}$

will be called the union sparsity pattern of the rows in matrix $C_{k j}$ which have non-zero elements in the active part of column $j$ $(j=k+1(1) n)$.

Theorem 2.1 The number of non-zero elements in matrix $S$ found by plane rotations does not depend on the permutation matrix $\underline{P}$ when the permutation matrix $Q$ is kept fixed.

Proof Assume that the multiplication with the fixed matrix Q has been performed before the beginning of the computations, i.e. $A_{1}=A Q$ and $A_{k}=\bar{A}_{k} \quad(k=1(1) n)$. It is clear that this assumption is not a restriction.

Consider the $k^{\prime} t h$ major step $(k=1(1) n)$. After the multiplication with any elementary orthogonal matrix $0_{\mu \nu} \quad\left(\mu \in M_{k k} \wedge\right.$ $v \in M_{k K}$ ) the sparsity patterns of the active parts of the rows in- 
volved in the computations are given by

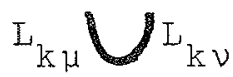

and

$\left(L_{k \mu} \cup L_{k \nu}\right) \backslash\{k\}$

where the second sparsity pattern is for the row in which a zero element in the pivotal column is produced. The expressions (2.8) show that after the last minor step in the $k^{\prime}$ th major step the sparsity patterns of the active parts of the rows involved are given by

$$
\bigcup_{i \in M_{k k}} L_{k i}=N_{k} \text { and }\left(\bigcup_{i \in M_{k k}} L_{k i}\right) \backslash\{k\}=N_{k} \backslash\{k\} \text {. }
$$

It is clear that the expressions in (2.9) do not depend on the order in which the zero elements are produced.

Consider now the sets $\mathbb{N}_{k j}^{*} \quad(j=k+1(1) n \wedge k<n)$. These sets are invariant during the computations at major step $k \quad(k=1(1) n-1)$. Indeed, new non-zero elements may be produced in some rows i ( $i \in M_{k k}$ ) but this happens only in the positions where there are non-zero elements in one or several of the other rows belonging to $M_{k k}$. Therefore $\mathbb{N}_{k, k+1}^{*}=\mathbb{N}_{k+1} \quad$ and $\quad \mathbb{N}_{k j}^{*}=\mathbb{N}_{k+1, j}^{*} \quad(j=k+2(1) n \quad \wedge$ $k+1<n)$.

Thus the theorem is proved because: (i) an arbitrary major step $k$ has been considered, (ii) the number, $\mathbb{N}_{k}$, of the nonzero elements in the $k^{\prime}$ th row of $\mathrm{S}$ does not depend on the order in which the zero elements in the pivotal column $\mathrm{k}$ are produced and (iii) the union sparsity pattern of the active parts of the rows which will be involved in the computations during the next major step, k+1'st, does not depend on the computations during the k'th major step. 
The following two results follow immediately from Theorem 2.1 .

Corollary 2.1 Assume that the same column interchâdes have been used both with Strategy 1 and Strategy 2. Then the number of non-zero elements in matrix. S will be the same for these two strategies.

口

Theorem 2.2 Assume that: (i) if at any major step there are several columns which have a minimal number of non-zero elements in their active parts, then the same column will be chosen as pivotal with both Strategy 1 and Strategy 2, (ii) after the column interchanges the number of elements in $M_{k k}$ is smaller than 4 for any $k \quad(k=1(1) n-1)$. Then the numbers of non-zero elements in $\mathrm{B}_{\mathrm{k}}$ found by Strategy 1 and Strategy 2 are the same for all $\mathrm{k}$.

Corollary 2.1 states that if the same matrix $Q$ is used, then Strategy 1 and Strategy 2 will produce matrices $S$ with the same number of non-zero elements. This does not mean, however, that the computational work needed to obtain (1.1) is also the same. The next example illustrates this.

Example 2.1 Consider the (14x8) matrix whose sparsity pattern is given in Fig. 2.1. The decomposition (1.1) has been performed with $Q=I$ and both Strategy 1 and Strategy 2 have been used to determine $P$ (it is readily seen that the use of $Q=I$ satisfies Rule 1 at each major step). The elementary orthogonal matrices which can be used at each major step are listed in Table 2.1 (the second index always shows the row where a zero element is pro- 
12 .

$$
\left[\begin{array}{llllllll}
x & x & 0 & 0 & x & 0 & 0 & 0 \\
0 & x & 0 & x & x & 0 & 0 & 0 \\
0 & 0 & x & 0 & 0 & 0 & x & 0 \\
0 & 0 & 0 & x & 0 & x & 0 & 0 \\
0 & 0 & 0 & 0 & x & 0 & x & 0 \\
0 & 0 & 0 & 0 & 0 & x & x & x \\
0 & 0 & x & 0 & 0 & 0 & 0 & x \\
x & x & 0 & x & 0 & 0 & 0 & x \\
0 & x & 0 & 0 & 0 & x & 0 & x \\
0 & 0 & x & 0 & x & 0 & 0 & x \\
0 & 0 & x & x & 0 & x & 0 & x \\
x & 0 & 0 & 0 & x & 0 & x & x \\
x & 0 & 0 & 0 & x & 0 & x & x \\
0 & 0 & x & 0 & 0 & x & 0 & 0
\end{array}\right]
$$

Fig 2.1

The sparsity pattern of a $(14 \times 8)$ matrix (the nonzero elements are denoted by $x$ in this figure)

duced). The numbers of minor steps and of fill-ins produced at each major step are given in Table 2.2. The total results show that even for this very small (but not very sparse) matrix the number of minor steps and fill-ins is reduced by about $7 \%$ when strategy 1 is used and when $Q$ is the same for both strategies. Of course, the numbers of non-zero elements in $S$ are the same, 33, for the two strategies.

Corolary 2.1 and Example 2.1 indicate that even when the same matrix $Q$ is used strategy 1 may be more profitable. One can expect that for large matrices $(n \geq 100)$ the reduction in minor steps and fill-ins will be greater than $7 \%$. However, if the matrix is extremely sparse, then Theorem 2.2 shows that both the storage and the computing time will be the same for the two strategies when $Q$ is the same and the sparsity is preserved well. Some ex- 


\begin{tabular}{|c|c|c|c|c|c|c|c|c|c|c|c|c|c|c|}
\hline \multicolumn{8}{|c|}{ Strategy } & \multicolumn{7}{|c|}{ Strategy 1} \\
\hline${ }^{2}$ & 1 & 2 & 3 & 4 & 5 & 6 & 7 & 1 & 2 & 3 & 4 & 5 & 6 & 7 \\
\hline 1 &, 8 & $0,1,12$ & $0_{1,13}$ & & & & & $0,1,8$ & $o_{12,13}$ & ${ }^{0} 1,12$ & & & & \\
\hline 2 & 9 & $\mathrm{O}_{2,8}$ & $\mathrm{O}_{2}, 12$ & 2,13 & & & & $0_{2,9}$ & $\mathrm{O}_{2,8}$ & $0_{2}, 12$ & & & & \\
\hline 3 &, 7 & $0_{3}, 14$ & $\mathrm{O}_{3,10}$ & 0,11 & & & & $O_{3,7}$ & $\mathrm{O}_{3,14}$ & $\mathrm{O}_{3,10}$ & 3,11 & & & \\
\hline 4 & & $0_{4,9}$ & $\mathrm{O}_{4,11}$ &,+ 12 & $D_{4,13}$ & & & $\mathrm{O}_{4,8}$ & $\mathrm{O}_{4,9}$ & $\mathrm{O}_{4,11}$ & 4,12 & & & \\
\hline 5 & & $\mathrm{O}_{5,9}$ & $0_{5,10}$ & & 5,12 & $0_{5,13}$ & & 0,13 & 8,9 & $0_{5,8}$ & 5,10 & 5,11 & $0_{5,12}$ & \\
\hline 6 & & ${ }^{0} 6,9$ & ${ }^{0} 6,10$ & 6,11 & 6,12 & 0,13 & 6,14 & ${ }^{0} 6,9$ & ${ }^{0} 6,8$ & ${ }^{0} 6,10$ & 6,11 & 6,12 & ${ }^{O_{6}, 13}$ & \\
\hline 7 &, 8 & $0_{7,9}$ & $\mathrm{O}_{7,10}$ & $D_{7,11}$ & $0,7,12$ & $O_{7}, 13$ & $0_{7,14}$ & $\mathrm{O}_{7}, 8$ & $0_{7,9}$ & $0_{7,10}$ & 7,11 & 7,12 & $O_{7,13}$ & $\mathrm{O}_{7,14}$ \\
\hline 8 &, 9 & $0_{0}, 10$ & $0_{8,11}$ & 8,12 & 8,13 & $0_{8,14}$ & & 0,9 & $0_{8,10}$ & 0,11 & 8,12 & 8,13 & $\mathrm{O}_{8,14}$ & \\
\hline
\end{tabular}

\section{Table 2.1}

The elementary orthogonal matrices used in the decomposition of the matrix from Fig. 2.1 $\left(\mathrm{k}=1(1) 8, i=1(1) \mathrm{s}_{\mathrm{k}}\right)$.

\begin{tabular}{|c|c|c|c|c|}
\hline \multirow{2}{*}{ Major } & \multicolumn{2}{|c|}{ Strategy 2} & \multicolumn{2}{|c|}{ Strategy } \\
\cline { 2 - 5 } $\mathrm{k}$ & Number of minor & Number of & Number of minor & Number of \\
\hline 1 & steps & fill-ins & steps & fill-ins \\
\hline 2 & 3 & 8 & 3 & 6 \\
3 & 4 & 8 & 3 & 7 \\
4 & 4 & 11 & 4 & 3 \\
5 & 5 & 3 & 4 & 3 \\
6 & 6 & 0 & 6 & 11 \\
7 & 7 & 0 & 6 & 0 \\
8 & 6 & 0 & 39 & 31 \\
\hline Total & 42 & 34 & 6 & 3 \\
\hline
\end{tabular}

Table 2.2

The number of minor steps and fill-ins found in the decomposition of the matrix from Fig.2.1 
14.

perimental results (Duff [7]) indicate that for very sparse matrices this is also true even when the requirement for the same $Q$ is removed.

In the real computations the matrices will not be extremely sparse (note that in [7] there are examples where the number of nonzero elements in the matrix is smalier than the number of rows) and Q will not be the same. In the next section some numerical experiments are carried out in order to compare the two strategies in such situations for matrices with large $n$. 


\section{Numerical results}

In this section a comparison of the performance of the two strategies will be carried out for some matrices with large $n$. Some information about the codes, the sparse technique and the test-matrices used is needed before the discusion of the numerical results which are given in Table 3.1 - Table 3.9.

3.1. The codes used The decomposition (1.1) is used for solving linear least-squares problems

(3.1) $\quad x=A^{+} b$

where $\mathrm{A}^{+}$is the pseudo-inverse $([18,19])$ of matrix A. Assume that paraliel computations with vector b are carried out during the decomposition (1.1) so that

$(3.2) \quad b^{*}=R^{T} \mathrm{~Pb}$

is available after the n'th major step. Then an approximation $x_{1}$ to $x$ can be found by

$$
x_{1}=Q S^{-1} D^{-1} b *
$$

Sometimes many problems (3.1) with the same matrix A have to be solved. In this case it is necessary to keep $R, D$ and $S$. When $A$ is dense $S$ is stored on and over the diagonal, while information about $R$ can be stored in an economical and stable way under the diagonal [23]. When matrix $A$ is sparse this is not efficient because matrix $R$ contains normally more non- 
16.

zero elements than matrix A. Therefore it is more profitable to store and keep a copy of the non-zero elements of $A$ [3,5]. If matrices $S, D$ and A are available, then an approximation $x_{1}$ (in general different from that computed by (3.3)) can be found from

$(3.4) \quad x_{1}=H^{T} \mathrm{~b}$

where

(3.5) $\quad H=Q_{Q}^{-1} D^{-2}\left(S^{T}\right)^{-1} Q^{T}$.

A special parameter T (drop-tolerance, see $[6,20,24,26])$ can be used during (1.1) so that if an element computed by (1.3) or (1.6) is smaller (in absolute value) than $T$, then this element is considered as a zero element in the further computations. Both storage and computing time may be saved when positive values of $T$ are used $([27,28,22,31])$. However, the use of $T>0$ may cause large errors in the approximation $x_{1}$ also. Therefore the following iterative process $(i=1(1) p-1)$

$$
r_{i}=b-A x, \quad \quad r_{i}^{*}=A^{T} r_{i},
$$

$$
\mathrm{d}_{i}^{*}=\operatorname{Hr} \underset{i}{*}
$$

$$
x_{i+1}=x_{i}+d_{i}^{*}
$$

has to be used when $T$ is large.

The combination, sparse matrix technique + Zarge drop-tolerance + iterative refinement, is used in the code LISSO1 (see 
$[29,30])$. The vectors $r_{i}, r_{i}^{*}, d_{i}^{*}$ and $x_{i}$ are stored in double precision. All inner products $(3.4)-(3.7)$ are accumulated in double precision. The non-zero elements of all matrices are stored in single precision. In this way the accepted approximation, $x_{p}$, as a rule has accuracy of order $O\left(\varepsilon^{2}\right)$ where $\varepsilon$ is the machine accuracy in single precision $[1,2,4]$. Strategy 1 is implemented in LLSS01. Another version, LISS02, where strategy 2 is implemented, has been developed. Since the only difference between LISSO1 and LISSO2 is the pivotal strategy, any difference in the results is caused by the pivotal strategy only.

3.2. Storage scheme. The storage scheme is the same for both codes and is based on ideas proposed by Gustarson [14,15]. Let NZ be the number of non-zero elements in A. Before the beginning of the computations the non-zero elements of matrix A are stored in the first $\mathrm{NZ}$ locations of a real array $A$. The order of the non-zero elements can be arbitrary but if $A(L)=a_{i j}$ then $\operatorname{SNR}(L)=j$ and $R N R(L)=i$ must be assigned $(L=1(1) N Z, \quad S N R$ and RNR are integer arrays of length NN). The codes order the nonzero elements by rows and store them in the first NZ locations of array A again, so that $A(L)=a_{i j} \wedge A(M)=a_{s t} \wedge i<s \Rightarrow L<M$ and $A(L)=a_{i j} \Rightarrow \operatorname{SNR}(L)=j$. Some additional information about the row starts and row ends is also stored by the codes. A copy of array $A$ and array $S N R$ is made in the real array $A 1$ and integer array SN (both of length NZ). The information about the row starts and row ends is also copied. This information is used in the iterative process ( $\operatorname{see}(3.6))$. In the pivotal search it is necessary to scan the columns. Therefore the structure is ordered by columns, but only the row numbers of the non-zero elements so ordered are stored before the beginning of the decomposition in the first NZ loca- 


\begin{tabular}{|c|c|c|c|c|c|c|c|c|}
\hline \multirow[b]{2}{*}{$\mathrm{m}$} & \multicolumn{4}{|c|}{$\mathrm{s}$ t $\mathrm{r}$ a $\mathrm{t}$ e $\mathrm{g} \quad \mathrm{y} \quad 1$} & \multicolumn{4}{|c|}{$\mathrm{s}$ t $r$ a $t$ e $g$ y 2} \\
\hline & COUNT & Time & Iterations & Accuracy & COUNT & Time & Iterations & Accuracy \\
\hline 100 & 210 & 0.58 & 5 & 4. $16 E-26$ & 210 & 0.60 & 5 & $1.91 E-26$ \\
\hline 110 & 220 & 0.73 & 7 & $1.06 \mathrm{E}-27$ & 227 & 0.82 & 9 & $2.88 \mathrm{E}-26$ \\
\hline 120 & 230 & 0.62 & 5 & $3.77 E-27$ & 230 & 0.65 & 5 & $3.51 \mathrm{E}-27$ \\
\hline 130 & 240 & 0.64 & 5 & $3.74 \mathrm{E}-27$ & 240 & 0.64 & 5 & $8.48 E-27$ \\
\hline 140 & 250 & 0.68 & 6 & $2.88 \mathrm{E}-27$ & 250 & 0.74 & 7 & $2.55 \mathrm{E}-27$ \\
\hline 150 & 260 & 0.65 & 5 & $3.74 E-27$ & 260 & 0.71 & 6 & $6.21 \mathrm{E}-27$ \\
\hline 160 & 270 & 0.77 & 7 & $1.54 E-27$ & 270 & 0.67 & 5 & $5.96 E-27$ \\
\hline 170 & 280 & 0.71 & 6 & $2.88 \mathrm{E}-27$ & 280 & 0.84 & 8 & $1.82 E-27$ \\
\hline 180 & 290 & 0.72 & 6 & $3.10 E-27$ & 290 & 0.67 & 5 & $5.35 \mathrm{E}-27$ \\
\hline 190 & 300 & 0.85 & 7 & $1.06 \mathrm{E}-27$ & 300 & 0.96 & 9 & $2.88 \mathrm{E}-27$ \\
\hline 200 & 310 & 0.83 & 6 & $3.69 \mathrm{E}-27$ & 310 & 0.97 & 8 & $1.74 \mathrm{E}-27$ \\
\hline
\end{tabular}

Table 3.1

Problems with matrices $A=F 2(m, 100,11,1,10)$ are solved. $N Z=m+110$, $N N=4800, T=10^{-25}$. The experiment has been run on a CDC Cyber 173.

tions of array RNR so that if $R N R(L)=i$ and $R N R(M)=s$ (where $i$ and $s$ are the row numbers of $a_{i j}$ and $a_{s t}$ ) and if $j<t$, then $I<M$. The row numbers of the non-zero elements in column $k$ are not needed after the major step $k$ and are therefore removed from array RNR by the codes. The non-zeroelements $a_{i k}$ ( $i>k$ ) are transformed into zero in major step $k$, therefore these elements and their column numbers are removed from arrays $A$ and SNR. Some elements (with their row and column numbers) may be removed when positive values of the drop-tolerance $T$ are used. Unfortunately, some new non-zero elements (fill-ins) are also created (when one of the elements involved in (1.6) is zero, while the other is non-zero). Such new elements and their column numbers (row numbers) are stored at the end of the row (column) if there are free lo- 


\begin{tabular}{|c|c|c|c|c|c|c|c|c|c|}
\hline \multirow{2}{*}{$m$} & \multicolumn{3}{|c|}{$\mathrm{S} t \mathrm{r}$ t $\mathrm{e} \mathrm{g} \mathrm{y}$} & \multicolumn{3}{|c|}{$\mathrm{S} \mathrm{t}$ a $\mathrm{t} \mathrm{g} \mathrm{y}$} \\
\cline { 2 - 9 } & COUNT & Time & Iterations & Accuracy & COUNT & Time & Iterations & Accuracy \\
\hline 100 & 872 & 1.42 & 5 & $4.53 \mathrm{E}-26$ & 878 & 1.60 & 7 & $3.04 \mathrm{E}-26$ \\
110 & 559 & 1.23 & 7 & $9.59 \mathrm{E}-26$ & 568 & 1.45 & 10 & $4.97 \mathrm{E}-27$ \\
120 & 522 & 1.18 & 7 & $2.90 \mathrm{E}-27$ & 526 & 1.27 & 8 & $2.20 \mathrm{E}-27$ \\
130 & 536 & 1.20 & 7 & $1.89 \mathrm{E}-27$ & 560 & 1.48 & 10 & $6.94 \mathrm{E}-27$ \\
140 & 611 & 1.15 & 5 & $1.96 \mathrm{E}-26$ & 617 & 1.33 & 7 & $8.78 \mathrm{E}-27$ \\
150 & 677 & 1.54 & 8 & $9.59 \mathrm{E}-28$ & 653 & 1.50 & 8 & $6.79 \mathrm{E}-27$ \\
160 & 638 & 1.42 & 7 & $1.62 \mathrm{E}-26$ & 687 & 1.58 & 8 & $6.21 \mathrm{E}-27$ \\
170 & 717 & 1.75 & 9 & $2.32 \mathrm{E}-27$ & 711 & 1.56 & 7 & $6.41 \mathrm{E}-27$ \\
180 & 776 & 1.73 & 7 & $9.59 \mathrm{E}-28$ & 783 & 1.80 & 8 & $1.01 \mathrm{E}-27$ \\
190 & 811 & 1.84 & 7 & $1.32 \mathrm{E}-26$ & 812 & 2.00 & 9 & $1.56 \mathrm{E}-26$ \\
200 & 817 & 1.73 & 5 & $3.02 \mathrm{E}-26$ & 858 & 2.10 & 9 & $9.69 \mathrm{E}-26$ \\
\hline
\end{tabular}

\section{Table 3.2}

Problems with matrices $A=F 2(m, 100,11,2,10)$ are solved. $N Z=2 m+110$, $N N=4800, T=10^{-25}$. The experiment has been run on a CDC Cyber 173.

cations. Otherwise a copy of the row (column) is made at the end of arrays $A$ and $S N R(R N R)$. It is clear that there is a limit to the number of new copies that can be made without exceeding the capacity of the arrays. Therefore occasional "garbage" collections are necessary. The "garbage" collections increase the computing time and should be avoided (using a large length for the arrays A, SNR and RNR) when this is possible. See more details about the storage scheme in [30].

3.3. Test-matrices. The results (especially the storage and the computing time) depend on the preservation of sparsity during (1.1). The preservation of sparsity depends on the dimensions of matrix A, on the distribution of the non-zero elements within A, 


\begin{tabular}{|c|c|c|c|c|c|c|c|c|}
\hline \multirow[b]{2}{*}{$\mathrm{m}$} & \multicolumn{4}{|c|}{$S$ t $r$ a $t$ e $g$ g $y$} & \multicolumn{4}{|c|}{$S t r a t e g y$} \\
\hline & COUNT & Time & Iterations & Accuracy & COUNT & Time & Iterations & Accuracy \\
\hline 100 & 1997 & 5.26 & 15 & $2.73 E-27$ & 2076 & 4.70 & 10 & $1.57 \mathrm{E}-27$ \\
\hline 110 & 2217 & 4.64 & 7 & $3.89 \mathrm{E}-27$ & 2439 & 5.76 & 7 & $2.68 \mathrm{E}-27$ \\
\hline 120 & 2331 & 4.89 & 5 & $1.34 E-26$ & 2095 & 5.09 & 8 & $1.97 \mathrm{E}-27$ \\
\hline 130 & 2336 & 5.96 & 9 & $7.57 E-26$ & 2378 & 6.50 & 9 & $2.02 \mathrm{E}-27$ \\
\hline 140 & 2287 & 5.94 & 7 & $5.58 E-27$ & 2476 & 6.71 & 8 & $7.57 \mathrm{E}-28$ \\
\hline 150 & 2896 & 8.35 & 7 & $2 \cdot 12 E-27$ & 2504 & 6.66 & 5 & $1.27 E-26$ \\
\hline 160 & 2644 & 8.82 & 9 & $2.27 \mathrm{E}-27$ & 3052 & 11.48 & 10 & $8.58 \mathrm{E}-28$ \\
\hline 170 & 2676 & 8.73 & 7 & $4.04 \mathrm{E}-28$ & 3198 & 13.21 & 11 & 1. $36 E-27$ \\
\hline 180 & 3361 & 14.14 & 8 & $1.92 E-27$ & 3595 & 16.93 & 5 & $8.96 \mathrm{E}-27$ \\
\hline 190 & 3334 & 14.46 & 7 & $3.08 E-27$ & 3435 & 16.33 & 5 & $1.26 E-26$ \\
\hline 200 & 3690 & 16.83 & 7 & $3.34 E-27$ & 4143 & 22.64 & 8 & $2.63 E-27$ \\
\hline
\end{tabular}

Table 3.3

Problems with matrices $A=F 2(m, 100,11,3,10)$ are solved. $\quad N Z=3 m+110$, $\mathbb{N} N=4800, T=10^{-25}$. The experiment has been run on a CDC Cyber 173.

on the magnitude of the non-zero elements and on the number of the non-zero elements before the beginning of the decomposition. Therefore, it is desirable to develop a generator for test-matrices where one or several of the above characteristics can be specified and changed automatically. Such a generator (subroutine MATRF2) has been developed ( a full documentation is in preparation). This generator produces matrices of class $F 2(m, n, c, r, \alpha)$ where the parameters can be varied and have the following significance: (i) the number of rows in the desired matrix can be specified by $m$, (ii) the number of columns in the desired matrix can be specified by $n$, (iii) the positions of certain non-zero elements within the desired matrix can be specified by $c$, (iv) the number $\mathbb{N Z}$ of non-zero elements in the desired matrix can be specified by $r$ so that $N Z=r m+110$, 


\begin{tabular}{|c|c|c|c|c|c|c|}
\hline \multirow[b]{2}{*}{$\mathrm{T}$} & \multicolumn{3}{|c|}{$S t r$ a $t$ e $g$ y 1} & \multicolumn{3}{|c|}{$S t r a t e g y$} \\
\hline & COUNT & Time & Iterations & COUNT & Time & Iterations \\
\hline $10^{-25}$ & $2706(0.85)$ & $8.91(0.85)$ & $8.0(1.03)$ & 2854 & 10.54 & 7.8 \\
\hline $10^{-2}$ & $1169(0.71)$ & $3.72(0.75)$ & $16.8(1.10)$ & 1646 & 4.98 & $15 \cdot 3$ \\
\hline $10^{-1}$ & $816(0.65)$ & $3.32(0.72)$ & $22.2(1.02)$ & 1259 & 4.58 & 21.7 \\
\hline
\end{tabular}

Table 3.4

Problems with matrices $A=F 2(m, 100,11,3,10), \quad m=100(10) 200$, are solved. $N Z=3 m+110, N N=4800$. The average results (for the 11 problems solved with each value of the drop-tolerance $T$ ) are given in this table. The ratios of the characteristics obtained by Strategy 1 and the corresponding characteristics obtained by Strategy 2 are given in brackets. The experiment has been run on a CDC Cyber 173 .

(v) the magnitude of the non-zero elements in the desired matrix can be specified by a so that $\max \left(\left|a_{i j}\right|\right) / \min \left(\left|a_{i j}\right|\right)=10 \alpha^{2}, a_{i j} \neq 0$. The matrices of class $F 2(m, n, c, r, \alpha)$ were used in all experiments. All parameters were varied in quite large intervals; some of the results are given in Table 3.1 - Table 3.9.

\subsection{Comparison of the storage required by the two strategies.}

It is not so easy to compare the storage required by the two strategies because $\underbrace{\text { the optimal value of }}_{\mathbb{N N}}$ (the length of the three large arrays A, SNR, RNR ) is not known in advance. The number of non-zero elements in $S$ should not be used in the comparison (if the column interchanges happen to be the same, then the same number of nonzero elements in matrix $S$ will be produced by the two strategies, 


\begin{tabular}{|c|c|c|c|c|}
\hline \multirow[b]{2}{*}{$\mathrm{m}$} & \multicolumn{2}{|c|}{$S t r a t e g$ y 1} & \multicolumn{2}{|c|}{$S t r a t e g y \quad z$} \\
\hline & Growth iactor & Smallest element & Growth factor & Smallest element \\
\hline 100 & 6.50 & $6.21 E-2$ & $2.88 E+15$ & $2.98 \mathrm{E}-32$ \\
\hline 110 & 4.69 & $8.85 E-3$ & $1.05 \mathrm{E}+11$ & $4.07 \mathrm{E}-27$ \\
\hline 120 & $2 \cdot 36$ & $1.69 \mathrm{E}-2$ & $2 \cdot 34 E+17$ & $7 \cdot 12 \pm-26$ \\
\hline 130 & 1.46 & $1.31 \mathrm{E}-1$ & $5.94 E+8$ & $1.66 E-23$ \\
\hline 140 & 2.07 & $3.27 E-2$ & $2 \cdot 38 E+12$ & $2.32 E-27$ \\
\hline 150 & 1.12 & $1.97 E-2$ & $1.54 E+14$ & $5.13 E-30$ \\
\hline 160 & 2.41 & $3.88 E-2$ & $1.02 \mathrm{E}+9$ & $2.22 E-20$ \\
\hline 170 & 4.42 & $6.98 E-3$ & $1.75 E+11$ & $6.31 E-27$ \\
\hline 180 & 4.50 & $1.59 E-3$ & $1.63 E+8$ & $1.55 \mathrm{E}-19$ \\
\hline 190 & 6.52 & $5.45 E-4$ & $4.05 E+12$ & $1.73 E-27$ \\
\hline 200 & 4.14 & $2.65 E-3$ & $1.13 E+8$ & $1.04 E-19$ \\
\hline
\end{tabular}

Table 3.5

Problems with matrices $A=F 2(m, 100,11,3,10)$ are solved. $\quad \mathbb{Z} Z=3 m+110$, $\mathbb{N N}=4800, T=10^{-2}$. "Growth factor" is the ratio of the largest (in absolute value)element found in array A during any step of the decomposition and the largest (in absolute value) element in array $\mathrm{A}$ before the beginning of the decomposition. "smallest element" is the smallest element in matrix $D_{n+1}$. The experiment has been run on a CDC Cyber 173.

but this does not mean that the computational work and the number of fill-ins are the same; see Corollary 2.1 and Example 2.1). Denote by COUNT, and $\operatorname{COUNT}_{2}$ the maximal numbers of nonzero elements kept in array $A$ when Strategy 1 and Strategy 2 are used. COUNT, and COUNT $_{2}$ can be used in the comparison (note that COUNT without any index will be used when there is no danger of misunderstanding). If e.g. $\operatorname{COUNT}_{1}<\mathrm{COUNT}_{2}$ on a given class of problems, then strategy 1 performs better than Strategy 2 for 


\begin{tabular}{|c|c|c|c|c|c|c|c|c|}
\hline \multirow[b]{2}{*}{$\mathrm{n}$} & \multicolumn{4}{|c|}{$S t r$ a $t$ e $g$ y 1} & \multicolumn{4}{|c|}{$S$ t $r$ a $t$ e $g$ y 2} \\
\hline & COUNT & Time & Iterations & Accuracy & COUNT & Time & Iterations & Accuracy \\
\hline 100 & 4323 & 2.95 & 9 & 1. $35 \mathrm{E}-14$ & 4685 & 3.82 & 9 & $1.67 E-14$ \\
\hline 110 & 4686 & 3.78 & 10 & $1.80 E-14$ & 4607 & 3.72 & 11 & $2.22 E-14$ \\
\hline 120 & 3212 & 1.15 & 9 & $2 \cdot 33 E-14$ & 4220 & 2.48 & 10 & $1.35 E-14$ \\
\hline 130 & 3160 & 1.22 & 11 & $1.60 E-14$ & 4144 & 2.25 & 9 & $1.87 E-14$ \\
\hline 140 & 2946 & 1.23 & 9 & $2.98 \mathrm{E}-14$ & 4145 & 1.92 & 7 & $1.82 E-14$ \\
\hline 150 & 3683 & 1.43 & 8 & $2.38 E-14$ & 4202 & 2.14 & 8 & $2.35 E-14$ \\
\hline
\end{tabular}

\section{$\underline{\text { Table } 3.6}$}

Problems with matrices $A=F 2(200, n, 11,6,10)$ are solved. $N Z=1110$, $\mathbb{N N}=10000, T=10^{-2}$. The experiment has been run on an IBM 3033.

this class because either smaller values of NN could be specified with Strategy 1 (reducing the storage) or the number of "garbage" collections with strategy 1 will often be smaller when the same values of $N N$ are used with both strategies.

Denote $\quad \mathrm{E}_{\mathrm{S}}=\operatorname{COUNT}_{1} / \mathrm{COUNT}_{2} \cdot \mathrm{E}_{\mathrm{S}}$ will be called the efficiency factor with regard to the storage requirements. By the use of Es the following conclusions can be drawn for the two strategies from our experiments with matrices of class $\mathrm{F} 2(\mathrm{~m}, \mathrm{n}, \mathrm{c}, \mathrm{r}, \alpha)$ (which were run with different values of the parameters).

(i) If the matrix is very sparse, then $E_{S} \approx 1$ is normally observed (see Table 3.1 , Table 3.2 and the first line of Table 3.8 ). This result should be expected (because in this situation it is very likely that the conditions of Theorem 2.2 are satisfied) and has already been reported in [7].

(ii) If the matrix is not very sparse, then often $E_{\mathrm{S}}<1$ (i.e. Strategy 1 performs better than Strategy 2), see Table 3.3 , Table 3.6 , Table 3.7 and Table 3.8 . 


\begin{tabular}{|c|c|c|c|c|c|c|c|c|c|}
\hline \multirow{4}{*}{$c$} & \multicolumn{3}{|c|}{$\mathrm{S} t \mathrm{r}$ a $\mathrm{e} g \mathrm{~g}$} & \multicolumn{2}{|c|}{$\mathrm{S} t \mathrm{t} \mathrm{t} \mathrm{e} \mathrm{g}$} & 2 \\
\cline { 2 - 9 } & COUNT & Time & Iterations & Accuracy & COUNT & Time & Iterations & Accuracy \\
\hline 20 & 4118 & 2.40 & 9 & $1.38 \mathrm{E}-14$ & 4088 & 2.30 & 7 & $1.07 \mathrm{E}-14$ \\
25 & 3975 & 2.50 & 8 & $3.94 \mathrm{E}-15$ & 4101 & 2.50 & 10 & $1.51 \mathrm{E}-14$ \\
30 & 3683 & 2.24 & 11 & $2.80 \mathrm{E}-14$ & 4088 & 2.33 & 10 & $2.42 \mathrm{E}-14$ \\
35 & 4033 & 2.57 & 8 & $2.93 \mathrm{E}-14$ & 4525 & 2.87 & 8 & $3.33 \mathrm{E}-14$ \\
40 & 3441 & 2.04 & 9 & $3.31 \mathrm{E}-14$ & 4560 & 3.13 & 8 & $3.20 \mathrm{E}-14$ \\
45 & 2612 & 1.26 & 9 & $3.09 \mathrm{E}-14$ & 3407 & 1.70 & 8 & $1.95 \mathrm{E}-14$ \\
50 & 3025 & 1.57 & 10 & $2.93 \mathrm{E}-14$ & 3840 & 1.94 & 10 & $2.64 \mathrm{E}-14$ \\
55 & 3478 & 1.92 & 10 & $2.64 \mathrm{E}-14$ & 4288 & 2.66 & 10 & $2.78 \mathrm{E}-14$ \\
\hline
\end{tabular}

\section{Table 3.7}

Problems with matrices $A=F 2(150,100, c, 6,10)$ are solved. NZ=1010, $N N=10000, T=10^{-2}$. The experiment has been run on an IBM 3033 .

(iii) If the drop-tolerance $T$ is increased and if the matrix is not very sparse, then $E_{S}$ becomes smaller (see Table 3.4). An explanation of this phenomenon can be given as follows. The algorithm described by $(1.7)-(1.11)$ cannot be applied with Strategy 2. This leads to very small elements in $D_{n+1}$ and very large elements in $A_{k}(k=1(1) n)$ when strategy 2 is used in the decomposition (1.1). Let $a=\max _{i, j}\left(\left|a_{i j}\right|\right)$ where $a_{i j} \quad(i=1(1) m, j=1(1) n)$ are the elements of matrix A before the beginning of the decomposition. Let $\bar{a}$ be the absolute value of the largest in absolute value element kept in array A during any step of the decomposition (1.1). The ratio $\bar{a} / a$ is called the growth factor. The results given in Table 3.5 show that the growth factors are very large when Strategy 2 is used (note that the growth factors become larger for $T=10^{-25}$; this shows that the growth factors tend to increase when the drop-tolerance $T$ is decreased). There- 


\begin{tabular}{|c|c|c|c|c|c|c|}
\hline \multirow[b]{2}{*}{$r$} & \multicolumn{2}{|c|}{$S t r a t e g y$} & 1 & \multicolumn{3}{|c|}{$\mathrm{S} t \mathrm{r} a \mathrm{t} \mathrm{e} \mathrm{g} \mathrm{y}$} \\
\hline & COUNT & Time & Iterations & COUNT & Time & Iterations \\
\hline 2 & $427(0.96)$ & $1.62(1.02)$ & $16.7(1.06)$ & 447. & 1.59 & $15 \cdot 7$ \\
\hline 3 & $816(0.65)$ & $3.32(0.72)$ & $22.7(1.05)$ & 1259 & 4.58 & 21.7 \\
\hline 4 & $1346(0.68)$ & $4.76(0.66)$ & $20.1(1.00)$ & 1989 & 7.16 & 20.2 \\
\hline 5 & $1962(0.72)$ & $7.96(0.71)$ & $21.6(1.03)$ & 2718 & 11.28 & 21.0 \\
\hline 6 & $2144(0.70)$ & $8.94(0.66)$ & $21.3(1.22)$ & 3044 & 13.47 & 17.4 \\
\hline
\end{tabular}

\section{Table 3.8}

Problems with matrices $A=F 2(m, 100,11, r, 10), \quad m=100(10) 200$, are solved. $N Z=r m+110, N N=4800, T=10^{-1}$. The average results (for the 11 problems solved with each value of parameter $r$ ) are given in this table. The ratios of the characteristics obtained by Strategy 1 and the corresponding characteristics obtained by strategy 2 are given in brackets. The experiment has been run on a CDC Cyber 173 .

fore the number of non-zero elements which are removed by the droptolarance is smaller for strategy 2.

It is necessary to emphasize here that the use of iterative refinement with a large value of the drop-tolarance gives a great reduction in storage for these problems (the use of two extra arrays A 1 and $S N$ in the iterative process is fully compensated because the length of the other three large arrays can be chosen much smaller when $T$ is large). This is normally so for problems which produce many fill-ins. If the problem does not produce many fill-ins ( if e.g. $r=1$ or $r=2$ ), then the use of iterative refinement will require some extra storage. 
26.

\begin{tabular}{|c|c|c|c|c|c|c|}
\hline \multirow[b]{2}{*}{$\alpha$} & \multicolumn{2}{|c|}{$\mathrm{s} t r a t e g \mathrm{y}$} & 1 & \multicolumn{2}{|c|}{$s t r a t$ e $g$ y } & 2 \\
\hline & $m=100$ & $m=150$ & $m=200$ & $m=100$ & $m=150$ & $\mathrm{~m}=200$ \\
\hline 10 & $4.16 \mathrm{E}-26$ & $2.88 E-27$ & $1.06 E-27$ & $1.96 \mathrm{E}-26$ & $6.21 \mathrm{E}-27$ & $1.74 \mathrm{E}-27$ \\
\hline $10^{2}$ & $4.38 E-25$ & $3.33 E-26$ & $2.29 E-26$ & $9.08 \mathrm{E}-26$ & $3.07 E-26$ & $3.50 \mathrm{E}-26$ \\
\hline $10^{3}$ & $2.20 E-24$ & $2.24 E-25$ & $2.01 E-24$ & $2.04 \mathrm{E}-24$ & $5.46 \mathrm{E}-25$ & $2.36 g-25$ \\
\hline $10^{4}$ & 1. $17 \mathrm{E}-23$ & $1.53 \mathrm{E}-24$ & $4.55 E-24$ & 1. $66 \mathrm{E}-23$ & 1. $15 \mathrm{E}-24$ & $6.97 \mathrm{E}-25$ \\
\hline $10^{5}$ & $4.93 E-23$ & $2.32 E-23$ & $8.45 E-24$ & $3.10 \mathrm{E}-23$ & $3.83 E-24$ & $2.12 \mathrm{E}-23$ \\
\hline $10^{6}$ & $1.06 \mathrm{E}-21$ & $2.00 \mathrm{E}-22$ & $1.05 E-22$ & $3.78 E-22$ & $2.64 \mathrm{E}-22$ & $3.70 E-22$ \\
\hline $10^{7}$ & $3.60 E-21$ & $1.24 E-21$ & $1.31 E-21$ & $2.63 E-21$ & $1.50 E-21$ & $2.18 \mathrm{E}-21$ \\
\hline $10^{8}$ & $1.60 \mathrm{E}-19$ & $3 \cdot 38 \mathrm{E}-20$ & 1. $11 \mathrm{E}-19$ & $5.77 \mathrm{E}-20$ & $1.22 E-19$ & 4. $55 E-19$ \\
\hline $10^{9}$ & $3.81 \mathrm{E}-17$ & $4.48 E-17$ & $1.46 \mathrm{E}-17$ & $8.44 E-17$ & $4.79 E-17$ & $1.79 E-3$ \\
\hline $10^{10}$ & $2.39 E-13$ & $7 \cdot 21 \mathrm{E}-16$ & $2.68 E-16$ & $3.66 \mathrm{E}-15$ & 1. $11 \mathrm{E}-13$ & $2.90 \mathrm{E} \quad 0$ \\
\hline
\end{tabular}

\section{Table 3.9}

Problems with matrices $A=F 2(m, 100,11,2, \alpha)$ are solved. $N Z=2 m+110$, $N N=4800, T=10^{-25}$. The accuracy of the approximations calculated by the two codes is given in this table. The experiment has been run on a CDC Cyber 173.

$$
\text { (iv) If } r<3, \text { then } \mathrm{E}_{\mathrm{S}} \approx 1 \text {. If } r \geq 3 \text {, }
$$

then $E_{S} \approx 0.7 ;$ see Table 3.8. No clear relation between $E_{S}$ and any of the parameters $m, n, c$ and $a$ has been observed; see some results in Table 3.3 , Table 3.6 and Table 3.7 . However, note that if the matrix is not very sparse, then strategy 1 performs better than strategy 2 for any choice of the parameters $m, n, c$ and $\alpha$.

3.5. Comparison of the computing time required by the two strategies. Denote by $t_{1}$ the computing time used by LLSSO1 and by $t_{2}$ that used by LLSS02. The number $E_{t}=t_{1} / t_{2}$ will be called the efficiency factor with regard to the computing time required. Conclusions (i) - (iv) from section 3.4 hold also when 
$E_{S}$ is replaced by $E_{t}$. This means that if the matrix is not very sparse, then the computing time will very often be reduced when Strategy 1 is used instead of Strategy 2. In some examples the computing time obtained by Strategy 1 is halved in comparison with that obtained by Strategy 2. See e.g. Table 3.6.

Note again the great efficiency of the use of large values of the arop-tolerance T (see Table 3.4). However, it should be noted that if the number of fill-ins is small (which is typical for very sparse matrices), then the use of a large drop-tolerance will cause more iterations and therefore extra computing time. of course, the use of large values for the drop-tolerance leads to extra iterations also when the matrix is not very sparse and many fill-ins are produced. However, the reduction in the computing time for the decomposition is so great in this case (because many fill-ins are removed by the droptolerance) that the total computing time is also reduced considerably.

\subsection{Comparison of the accuracy achieved by the two strategies.} If the matrix is well conditioned (if $\alpha<100$ in our experiments), then the accuracy achieved by the two subroutines is approximately the same (of order of magnitude $O\left(\varepsilon^{2}\right)$ where $\varepsilon$ is the machine accuracy; $\varepsilon=0\left(10^{-14}\right)$ for CDC Cyber 173 and $\varepsilon=0\left(10^{-7}\right)$ for IBM 3033). The fact that the accuracy achieved has order of magnitude $O\left(\varepsilon^{2}\right)$ is in full agreement with the results obtained by Björck $[1,2,4]$. Note that the number of iterations needed to obtain such accuracy is approximately the same for both subroutines when the drop-tolerance is very small, $T=10^{-25}$ in our experiments. ( in Table 3.1 and Table 3.2 the numbers of iterations for strategy 1 are slightly smaller than those for strategy 2 ). There is a tendency that the numbers of iterations for strategy 2 are smaller than those for Strategy 1 when the drop-tolerance is 
large (see Table 3.4 and Table 3.8 ). This is probably caused by the fact that more elements are removed when strategy 1 is used with a large drop-tolerance ( see the values of count in the tables and Section 3.4) and thus the decomposition computed by strategy 1 is not very accurate. Since the computational cost per iteration is much smaller than that for the decomposition (1.1), the fact that Strategy 1 uses more iterations has no visible influence on the efficiency factor with regard to the computing time required (see Section 3.5).

An experiment with different values of a has also been carried out. The matrices $A=F 2(m, 100,11,2, \alpha)$ with $m=100(10) 200$ have been used in this experiment with $T=10^{-25}$ and $\alpha=10(10) 10^{10}$. The results for $\alpha<10^{9}$ are comparabie. For $\alpha=10^{9}$ and $\alpha=10^{10}$ Strategy 1 gives much more accurate results for some problems. The results for $m=100, m=150$ and $m=200$ are given in Table 3.9 .

The experiments indicate that the fact,that Strategy 2 produces very small elements in $D_{n+1}$ and very large (in absolute value) elements in $A_{k} \quad(k=1(1) n+1)$, has not a great influence on the accuracy of the approximations computed using this pivotal strategy

\subsection{Robustness of the computations. The fact, that the elements} of $D_{n+1}$ become very small and the growth factors (see section 3.4) become very large when Strategy 2 is used (see Table 3.5), can lead to underflows and overflows. Note that, when the problem with matrix $A=F 2(110,100,11,2,10)$ was solved with strategy 2 , the growth factor was of magnitude $O\left(10^{140}\right)$. On CDC Cyber 173 this did not cause trouble, but on many other computers such a large growth factor will lead to overflow. Of course, there is no guarantee against overflows when strategy 1 is used. But the results given in Table 3.5 
indicate that the computations with strategy 1 are much more robust than those with strategy 2 .

3.8. General conclusion. The numerical experiments indicate that Strategy 1 should bepreferred in the choice between the two strategies discussed in this paper when problem (3.1) is solved and when the plane rotations have to be used in the decomposition of matrix A. 
1. A. Björck, Iterative Refinement of Linear Least Squares Problems I. BIT 7, 257-278 (1967).

2. A. Björck, Iterative Refinement of Linear Least Squares Problems II. BIT 8, 1-30 (1968).

3. A. Björck, Methods for Sparse Linear Least Squares Problems. In Sparse Matrix Computations (Edited by J. Bunch and D. Rose), pp. 179-199. Academic Press, New York $(1976)$.

4. $\AA$. Björck, Comment on the Iterative Refinement of Least Squares Problems. J.Amer. Stat. Assoc. 73, 161-166 (1978).

5. A. Björck, Numerical Algorithms for Linear Least Squares Problems. Report No. 2/78. Department of Mathematics, University of Tronaheim, Trondheim, Norway (1978).

6. R. J. Clasen, Techniques for Automatic Tolerance in Iinear Programming. Comm. ACM 9, 802-803 (1966).

7. I. S. Duff, Pivot Selection and Row ordering in Givens Reduction of Sparse Matrices. Computing 13, 239-248 (1974).

8. I. S. Duff and J. K. Reid, A Comparison of Some Methods for the Solution of Sparse overdetermined sistems of Linear Equations. J. Inst. Math. Appl. 17, 267-280 (1976). 
9. W. M. Gentleman, Least Squares Computations by Givens' Transformations Without Square Roots. J. Inst. Math. Appl. $12, \quad 329-336(1973)$.

10. W. M. Gentleman, Row EZimination for Solving Sparse Linear Systems and Least Squares Problems. In Numerical Analysis Dundee 1975 (Edited by G. A. Watson), pp. 122-133. Lecture Notes in Mathematics No 506, Springer, Berlin (1975).

11. W. M. Gentleman, Error Analysis of QR Decompositions by Givens' Transformations. Iin. Alg. Appl. 10, 189-197 (1975).

12. J.W. Givens, Numerical Computation of the Characteristic Values of a Real Symmetric Matrix. Report No. ORNL-1574. Oak Ridge National Laboratory (1954).

13. J.W. Givens, Computations of Plane Unitary Rotations Transforming a General Matrix to Triangular Form.

J. Soc. Ind. Appl. Math. 6, 26-50 (1958).

14. F. G. Gustavson, Some Basic Techniques for Solving sparse Systems of Linear Equations. In Sparse Matrices and Their Applications (Edited by D. J. Rose and R. A. WiIloughby), pp. 41-52. Plenum Press, New York (1972).

15. F. G. Gustavson, Two Fast Algorithms for Sparse Matrices: Multiplication and Permuted Transposition. ACM Trans. Math. Software 4, 250-269 (1978). 
16. S. Hamarling, A Note on Multiplications to Givens Plane Rotations. J. Inst. Math. Appl.13, 215-218 (1974).

17. C. L. Lawson, R. J. Hanson, D. R. Kinsaid and F. T. Krogh, Basic Linear Algebra Subprograms for Fortran Usage. ACM Trans. Math. Software 5, 308-323 (1979).

18. E. Hoore, On the Reciprocal of the General Algebraic Matrix. Bull. Amer. Math. Soc. 26, 394-395 (1919-1920).

19. R. Penrose, A Generalized Inverse for Matrices. Proc. Cambridge Phil. Soc. 51, 506-513 (1955).

20. J.K. Reid, Fortran Subroutines for Handling Sparse Linear Programming Bases. Report No. R8269, A.E.R.E., Harwell, England (1976).

21. J.K. Reid, Sparse Matrices. In The State of the Art in Numerical Analysis (Edited by D. A. H. Jacobs), pp. 85-146. Academic Press, London (1977).

22. K. Schaumburg, J. Wasniewski and Z. Zlatev, The Use of Sparse Matrix Technique in the Numerical Integration of Stiff Systems of Linear Ordinary Differential Equations. Computers and Chemistry 4, to appear.

23. G. W. Stewart, The Economical Storage of Plane Rotations. Numer. Math. 25, 137-139 (1976).

24. R. P. Tewarson, Sparse Matrices. Academic Press, New York (1973) 
25. J.H. Wilkinson, Some Recent Advances in Numerical Iinear Algebra. In The state of the Art in Numerical Analysis (Edited by D.A.H.Jacobs), pp. 3-53. Academic Press, New York (1977).

26. P. Wolfe, Error in the Solution of Linear Programming Problems. In Error in Digital Computations (Edited by L.B.Rall), Vol.2, pp.271-284. Wiley, New York (1965).

27. Z. Zlatev, Use of Iterative Refinement in the Solution of Sparse Linear Systems. Report 1/79. Institute of Mathematics and Statistics, The Royal Veterinaxy and Agricultural University, Copenhagen, Denmark (1979).

28. Z. Zlatev, on Solving some Large Linear Problems by Direct Methods. Report 111. Computer Science Department, Aarhus University, Aarhus, Denmark (1980).

29. Z. Zlatev and H. B. Nielsen, LLSSOI - a Fortran Subroutine for Solving Lest Squares Problems (USER'S GUTDE). Report No. 79-07. Institute for Numerical Analysis, Technical University of Denmark, Lyngby, Denmark (1979).

30. Z. Zlatev and H. B. Nielsen, Least Squares Solution of Large Linear Problems. In Symposium i Anvendt Statistik 1980 (Edited by A.Höskuldsson,K.Conradsen,B.Sloth Jensen and K. Esbensen), pp.17-52. NEUCC, Lyngby, Denmark (1980).

31. Z. Zlatev, J. Wasniewski and K. Schaumburg, Comparison of Two Algorithms for Solving Large Linear Systems. Report 80/9. Regional Computing Centre at the University of Copenhagen, Copenhagen, Denmark (1980). 